\title{
Efficient growth techniques and properties of Benzophenone Single Crystals for NLO Applications:A Review
}

\author{
Selvakumar. P \\ GRD Centre for Materials Research, \\ PSG College of Technology, \\ Coimbatore, India
}

\author{
Arun Kumar. R \\ GRD Centre for Materials Research, \\ PSG College of Technology, \\ Coimbatore, India,
}

\author{
Arivanandhan. M \\ Shizuoka University, \\ Hamamatsu, Japan.
}

\author{
Hayakawa. Y \\ Shizuoka University, \\ Hamamatsu, Japan.
}

\author{
Kawai. $\mathrm{H}$ \\ Shizuoka University, \\ Hamamatsu, Japan.
}

\begin{abstract}
Benzophenone is one of the promising organic NLO materials for their high energy laser photonic applications and hence this review is mainly emphasized on the discussion of growth, efficiency and applications of low melting organic NLO benzophenone crystals in order to improve their structural and physical properties. To grow bulk and good quality benzophenone crystals by various techniques adopted has been discussed such as slow evaporation method, Microtube-Czochralski method, and Vertical Bridgman method. It is acknowledged that the size and the efficiency of the growing benzophenone single crystal varies with that of the techniques we are using has been discussed below. The SHG efficiency of pure benzophenone crystal also been compared with the standard KDP crystal. Also optical transparency behaviour of the benzophenone crystal has also been reviewed.
\end{abstract}

Keywords: Benzophenone, single crystals, Solution growth, Melt growth, NLO, SHG efficiency

\section{INTRODUCTION}

\subsection{Necessity for the organic NLO}

\section{Crystals:}

Purely inorganic NLO materials have excellent mechanical and thermal properties but possess relatively modest optical nonlinearity because of the lack of extended $\pi$-electron delocalization [1] but some organic NLO crystals form a non-centrosymmetric structure which exhibit second-order nonlinear optical properties that far surpassed those of the conventional materials has now led to the synthesis and evaluation of a wide range of potentially useful solid materials which have potential applications in signal transmission, data storage, optical switching, laser printing, displays, inflorescence, photolithography, remote sensing, chemical and biological species detection, high resolution spectroscopy, medical diagnosis and underwater monitoring \& communication [2]. Thus Organic NLO materials are often superior to inorganic in terms of their response speed, optical clarity and the magnitude of their third order susceptibility and for a material to exhibit NLO activity it should be non Centro symmetric. Hence it may be useful to prepare organic crystals which have aromatic rings having high non linear optical coefficient, higher laser damage threshold, fast response, low mobility and large band gap applications [3]. 


\subsection{Significance of the present review:}

Although, organic crystals with higher nonlinear optical (NLO) efficiency have the great importance for their applications in high-energy lasers applications but it is difficult to grow the bulk crystals of pure organic materials because of their low thermal stabilities and presence of large chromophoric cations and hence the organic crystals generally grow with structural defects. To realize the full efficiency of devices based on single crystals, crystals must be free from the defects since solution growth includes lot defects also it is difficult to grow large size organic single crystals and hence by adding suitable dopants and functional groups.

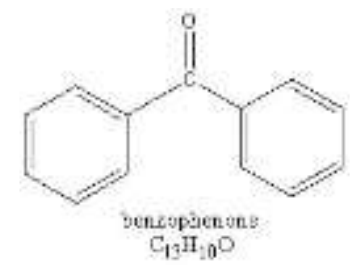

Figure 1. Structure of benzophenone.

we can modify the physical properties of single crystals which tends to enhance bulk growth of organic crystals with less defects but from some literature survey tells that even by adding such dopants and functional groups may hardly control the defects in crystals and hence by adopting various growth techniques results in improvement in molecular alignment of the crystals and tend to modify the crystal quality and in its perfection. Thus in this review the mainly focused discussion is on growing large and perfect benzophenone single crystals for high-energy laser applications since it is a promising NLO material where the absorption is visible in the blue light region having a cut-off wavelength lower than $450 \mathrm{~nm}$ $[4,5,6]$ and it has nearly six times higher NLO efficiency than that of Potassium dihydrogen phosphate (KDP), a well-known inorganic NLO material $[7,8]$.
Table 1. Crystal data of benzophenone.

\begin{tabular}{ll} 
Empirical formula : $\mathrm{C}_{13} \mathrm{H}_{10} \mathrm{O}$ \\
Formula weight & $: 182.217(\mathrm{~g} \cdot \mathrm{mol}-1)$ \\
Crystal system & $:$ orthorhombic \\
Space group & $: \mathrm{P} 22_{1} 2_{1}[9]$ \\
Boiling-point & $: 305 .{ }^{\circ} \mathrm{C}$ \\
Melting-point & $: 48.5^{\circ} \mathrm{C}(\alpha$ form $) \& 26^{\circ} \mathrm{C}$ \\
& $(\beta$ form $[10]$ \\
Density & $: 1.111$ at $18{ }^{\circ} \mathrm{C}$ \\
Vapour pressure & $: 1.93 \times 10-3 \mathrm{~mm} \mathrm{Hg}$ at $25{ }^{\circ} \mathrm{C}$ \\
Refractive index & $: 1.696$ at $19{ }^{\circ} \mathrm{C}[11]$ \\
Solubility & $:$ Practically insoluble in water \\
& but soluble in organic \\
& solvents such as alcohol, \\
& ether, acetone, acetic acid, \\
chloroform and & benzene $[12]$ \\
& $:>110{ }^{\circ} \mathrm{C}$ \\
Flash-point & $:$ Decomposes on heating to \\
Stability & produce toxic gases; reacts \\
& with strong oxidants. \\
\hline
\end{tabular}

\section{GROWTH TECHNIQUES:}

Since benzophenone is a promising organic NLO material due to its enormous applications [13], it is necessity to grow benzophenone with high quality and perfection crystals [14]. So from many literature surveys which had been discussed about various growth techniques such as indirect laser heated pedestal growth (ILHPG) method [15], MicrotubeCzochralski ( $\mu \mathrm{T}-\mathrm{CZ}$ ) method [16], seed-oriented undercooled melt growth [17], Vertical Bridgman (VB) method [18], and uniaxially solution crystallization (USC) method of Sankaranarayanan-Ramasamy [19] have been reported with the aim of growing bulk organic NLO benzophenone single crystals. Among all the above stated methods, the Microtube-Czochralski and Vertical Bridgman methods are more versatile, bulk and unidirectional crystals can be grown by optimizing the growth parameters [12]. 


\section{International Journal of Science and Engineering Applications Special Issue NCRTAM ISSN-2319-7560 (Online)}

Let we see how some of the techniques are adopted for the growth of benzophenone single crystals.

\subsection{Solution Growth:}

Growth of $\mathrm{NaCl}$ (common salt) from aqueous solutions (sea water) is considered as one of the ancient methods of solution growth in which crystals are grown by slow evaporation technique. The method of crystal growth from low temperature aqueous solution is extremely popular due to its easy way of production and technologically importance due to its cost effectiveness. It is the most widely used method for the growth of single crystals since from ancient time but the major disadvantage for growth of single crystal by low temperature solution growth may takes uncertainity period of time. It may be of weeks, months and sometimes years. Though the technology of growth of crystals from solution has been well perfected, it involves meticulous work, much patience and even a little amount of luck. An external impurity, power failure or a contaminated batch of raw material can destroy months of work.

\subsubsection{Slow evaporation technique:}

In this solution technique by Claude et al has grown the benzophenone single crystal by taking solid flaky benzophenone salt as precursor and dissolved in ample amount of alcohol till it reaches saturation state. The solubility and supersolubility of benzophenone was observed to be 10 grams and 15 grams per $100 \mathrm{ml}$ of ethanol solvent. Then the supersaturated solution is kept as constant temperature solution growth process for nucleation and subsequent crystallization. As grown crystals of benzophenone were a cleaved sample of size $4.5 \mathrm{~cm} \times 4.5 \mathrm{~cm} \times 4 \mathrm{~cm}$ was the crystal of the sample crystallized using solution growth apparatus [20].

\subsection{Melt Growth:}

In the literature the benzophenone single crystal was first grown by the Czochralski technique [21] followed by reports of benzophenone crystals were grown from the solution method [22] and and then by Bridgmann technique [23]. There are various types of melt growth but in accordance with the literature survey only suitable method for growing benzophenone single crystal were discussed below.

\subsubsection{Microtube-Czochralski ( $\mu$ T-CZ)}

growth:

The benzophenone crystal, an organic nonlinear material was grown by Czochralski method by Bleay et al. [21] for the first time which has high imperfection with smaller dimension. Tachibana et al. [24] has grown much better than the previous benzophenone crystals by using the similar experimental setup of Bleay et al. A technical brief of the experimental setup employed in this investigation can be found elsewhere [25]. The brief description of this technique is discussed here. Highly purified benzophenone material was filled in a circular shape static glass crucible. The source material filled crucible has to be placed inside a resistive heated furnace. Commercially bought Eurotherm temperature controller with an accuracy of \pm $0.1^{\circ} \mathrm{C}$ should be employed to control the temperature of the furnace. Instead of seeding by pre-grown defect free seed crystal, stainless steel micro tube of micrometre size $(\mu \mathrm{m})$ has to be used for seeding the melt. Since melt wets the inner walls of the fine capillary tube, it rose to a height, which depended by the tube radius, the surface tension of the melt, the melt density and the contact angle of the melt with micro tube. A fine column of melt raised inside the micro tube will be crystallized first due to heat dissipation through seed rod and the grown crystal inside the micro tube was acted as a seed for further growth. The growth temperature and the pulling rate of the crystal should be optimized for the growth of benzophenone single crystals. The optimizing growth parameters for the present investigation are, pulling rate: $1.0-1.5 \mathrm{~mm} / \mathrm{hr}$, seed rotation rate: $5-10 \mathrm{rpm}$, the cooling rate: $1^{\circ} \mathrm{C} \cdot \mathrm{hr}^{-1}$, length of the micro tube underneath the melt surface: $1.5 \mathrm{~mm}$ and the axial thermal gradient: $8^{\circ} \mathrm{C} / \mathrm{cm}$. Once the growth run was completed, the system temperature was reduced to room temperature $\left(31^{\circ} \mathrm{C}\right)$ at a predefined cooling rate to avoid the thermal stress in the grown crystal. 


\subsubsection{Vertical Bridgman (VB) growth:}

By the literature survey of (M. Arivanandhan et al.), bulk crystals of benzophenone were grown using the VB system. This VB system consists of three major parts such as transparent furnace, temperature controller and ampoule translation assembly. The transparent furnace consist a central quartz tube which is centrally placed in a glass beaker filled with two immiscible liquids. Sufficient volumes of deionized water and sunflower oil (normally used for cooking) were used for low temperature and high temperature zones respectively since the melting point of benzophenone is $\sim 48^{\circ} \mathrm{C}$. Spiral shaped tubular resistive heaters were encircled the growth tube at hot and cold zones. Commercially bought eurotherm temperature controller with an accuracy of $\pm 0.1^{\circ} \mathrm{C}$ was employed to control the zone temperatures. Direct observation of solidliquid interface, which is more feasible in transparent furnaces than conventional furnaces, is important for the directional solidification to determine the desired interface shape by controlling the growth parameters. In the case of VB growth of organic material, due to its low thermal conductivity one has to adopt the recommended translation rate of $1-2 \mathrm{~mm} \cdot \mathrm{h}^{-1}$ [26]. The more details of the growth processes can be found elsewhere [27].

\section{RESULTS AND DISCUSSIONS:}

In this section we discuss some of the literature surveys of results on the nonlinear optical properties of benzophenone single crystals which were grown by various techniques.

\subsection{Optical studies:}

Since for the grown benzophenone crystal to be used in optical applications should have high transmittance (\%), which had been given by (A. Claude et al.) He studied the absorption spectra of benzophenone which was taken UV-VIS spectrophotometer where two prominent absorption maxima were observed at $225 \mathrm{~nm}$ and $255 \mathrm{~nm}$ respectively. It also has two additional maxima, one at $285 \mathrm{~nm}$ and another at $345 \mathrm{~nm}$. A transmission peak at $255 \mathrm{~nm}$ signifies a $\pi$ to $\pi^{*}$ transition and identification of the presence of the benzene rings in the radical.
Aromatic systems, which contain $\mathrm{p}$ electrons, absorb strongly in the ultraviolet which fulfils the efficient properties of having high nonlinear optical coefficient, higher laser damage threshold, fast response, low mobility and large band gap and high energy laser applications [3].

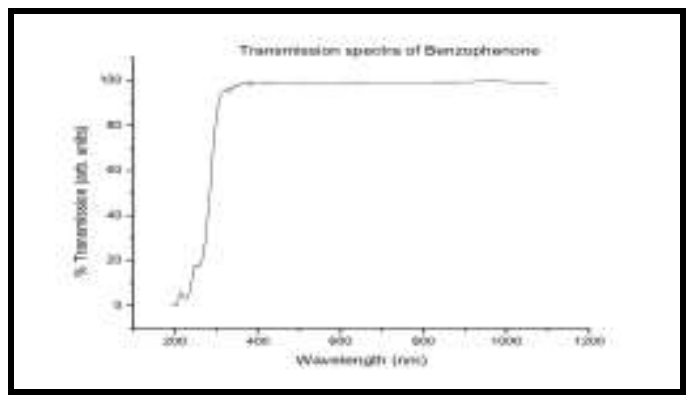

Figure 2. Transmission Spectra of
benzophenone
(adapted from $[20]$.

This is evident by a previous transmission peak at $245 \mathrm{~nm}$ and the very low transmission were observed below the cutoff wavelength and high transparency at 400-900 nm highlighted the optical property of the grown benzophenone crystal which is given below.

\subsection{SHG Efficiency:}

Powder SHG measurement by Kurtz method is an important and popular study to identify the conversion efficiency of grown benzophenone NLO material. The SHG efficiency of the benzophenone grown by MicrotubeCzochralski ( $\mu \mathrm{T}-\mathrm{CZ}$ ) method was given by $M$. Arivanandhan et al. The measured SHG efficiency by Kurtz powder method was found to be three times higher than that of the efficiency of the standard KDP crystals [28].

\section{CONCLUSION:}

As to conclude this review, we have noticed that in the near future, benzophenone crystal can be grown easily, bulky with high perfection as many recent reported works attracted the researchers by some unique advantage of growing bulk and unidirectional benzophenone crystals at ambient temperature which causes minimum thermal induced grown-in defects using 
many experimental set-up with high conversion efficiency and high growth rate.

\section{REFERENCES}

[1] Lakshmana perumal, C. K., et al., J. Cryst. Growth 240(2002)212.

[2] Tang, C.W., Van.Slyke, S.A., Appl.Phys.Lett.51 (1987)913-915

[3] Dalton, L.R., Pure Appl.Chem.76 (2004)1421-1433

[4] Chemla, D.S., and Zyss, J., (1987), 'Vol.1-2, Academic Press, Orlando, New York.

[5] Levine, B.F., Bethea, C.G., Thurmond, C.D., Lynch.R.T, Bernstein.J.L.(1979), J. Appl. Phys. 50, 2523.

[6] Kerkoc, P., Zgonic, M., Sutter, K., Bosshard, Ch., Gunter, P. (1989), Appl. Phys. Lett. 54, 2062.

[7] Lammers, D., Betzler, K., Xue, D., Zhao, J. Optical Second Harmonic Generation in Benzophenone. Physica status solidi (a) 2000; 180: R5-R7.

[8] Wang, W., Lin, X., Huang, W. Optical properties of benzophenone single crystal grown from undercooled melt with oriented growth method. Optical Materials 2007; 29: 1063- 1065 .

[9] Masaru Tachibana, Shigeki Motomura, Akira Uedono, Qi Tang and Kenichi Kojima. Characterization of grown-in dislocations in Benzophenone single crystals by X-ray Topography. Japanese Journal of Applied Physics 1992; 31: 2202-2205.

[10] Zhao, J., Yang, D., Zhang, Z., Photonic Acta 28, 280 (1998).

[11] Lammers, D., Betzler, K., Xue, D., and Zhao, J., Phys. stat. sol. (a) 180, R5 (2000).

[12] Direction Controlled Growth of Organic Single Crystals by Novel Growth Methods "Advanced Topics on Crystal Growth" Direction Controlled Growth of Organic Single Arivanandhan, M., Natarajan, V., Sankaranarayanan, K., and Hayakawa, Y., http://dx.doi.org/10.5772/53037.
[13] Bloembergen, N., Nonlinear Optics, Benjamin. New York 1965.

[14] Hammond, R. B., Pencheva, K., Roberts, K. J. An examination of polymorphic stability and molecular conformational flexibility as a function of crystal size associated with the nucleation and growth of benzophenone. Faraday Discuss 2007; 136: 91-106.

[15] Yokoo, A., Tomaru, S., Yokohama, I., Kobayashi, H., Itoh, H., Kaino, T. A new growth method for long rod-like organic nonlinear optical crystals with phase-matched directions. Journal of Crystal Growth 1995; 156: 279-284.

[16] Sankaranarayanan, K., Ramasamy, P. Microtube-Czochralski technique: a novel way of seeding the melt to grow bulk single crystal. Journal of Crystal Growth 1998; 193: 252-256.

[17] Wang, W., Huang, W., Ma, Y., Zhao, J. Oriented growth of benzophenone crystals form undercooled melts. Journal of Crystal Growth 2004; 270: 469-474.

[18] Arivanandhan, M., Sankaranarayanan, K., Ramamoorthy, K., Sanjeeviraja, C., Ramasamy, P. A novel may of modifying the thermal gradient in Vertical BridgmanStockbarger Technique and studies on its effects on the growth of benzophenone single crystals. Crystal Research and Technology 2004; 39: 692-698.

[19] Sankaranarayanan, K., Ramasamy, P. Unidirectional seeded single crystal growth from solution of benzophenone. Journal of Crystal Growth 2005; 280: 467-473.

[20] Thenmozhi, R., and Claude, A., Arch. Appl. Sci. Res., 2012, 4 (2):898-905.

[21] Bleay, J., Hooper, R. M., Narang, R.S. and Sherwood, J. N., J. Cryst. Growth, 43, (1978) 589.

[22] Hooper, R. M., McArdle, B. J., Narang, R. S., and Sherwood, J. N., in ed.: Pamplin, B., Crystal Growth, Pagamon Press, Oxford, p. 395 (1980). 


\section{International Journal of Science and Engineering Applications Special Issue NCRTAM ISSN-2319-7560 (Online)}

[23] McArdle, B. J., and Sherwood, J. N., in eds.: Dryburgh, P. M., Cockayne, B. and Barraclugh, K. G., Advanced Crystal Growth, Prentice Hall, New York, p. 179 (1987).

[24] Tachibana, M., Motomura, S., Uedono, A., Tang, Q., Kojima, K., Jpn. J. Appl. Phys.31 (1992) 2202.

[25] Sankaranarayanan, K., Ramasamy, P. Microtube-Czochralski technique: a novel way of seeding the melt to grow bulk single crystal. Journal of Crystal Growth 1998; 193:252-256.

[26] McArdle, B. J., Sherwood J. N., Damask, A. C. The growth and perfection of phenanthrene single crystals: 1 . Purification and single crystal growth. Journal of Crystal Growth 1994; 22: 193-200. 